\title{
CORRELATION FUNCTIONS FOR PAIRS AND GROUPS OF GALAXIES
}

\author{
M. Kalinkov and I. Kuneva \\ Department of Astronomy, Bulgarian Academy of Sciences \\ 72 Lenin blvd, 1784 Sofia, Bulgaria
}

\section{INTRODUCTION}

There are many studies on the correlation functions of galaxies, of clusters of galaxies, even of superclusters (e.g. Groth and Peebles 1977; Davies and Peebles 1983; Kalinkov and Kuneva 1985, 1986; Bahcall 1988 and references therein) but not so many on pairs and groups of galaxies.

Results of the calculations of two-point correlation functions for some catalogs of pairs and groups of galaxies are given in this paper. It is assumed that the distances to pairs and groups of galaxies are given by their mean redshifts according to $\mathrm{R}=\sum_{i=1}^{n} \mathrm{~V}_{i} / n \mathrm{H}_{0}$, where $n$ is the number of galaxies in the system and $\mathrm{H}_{0}=100 \mathrm{~km} \mathrm{~s}^{-1} \mathrm{Mpc}^{-1}$.

\section{METHOD}

The spatial correlation functions for the systems centers is determined from the relation,

$$
\xi_{p p, g r g r}=n_{0} /\left(n_{r}(r)-1\right)
$$

Here $n_{0}(r)$ is the number of systems, the centers of which are located in the interval $(r-\Delta r / 2, r+\Delta r / 2) ; n$ is the corresponding number for the objects from the random catalogs; $\xi_{p p}$ and $\xi_{g r g r}$ stand for pair-pair and group-group correlation functions.

For random catalog generation it is necessary to reproduce all selections applied to the observed sample. The method used here is as follows. The apparent distribution of the system centers in the Northern and Southern galactic hemispheres, more often in elementary cells $\Delta l \times \Delta b=10^{\circ} \times 5^{\circ}$, were investigated. In each strip along $l$, for a given $b$ there is a test for which cells the counts of the systems centers may be regarded as uniformly distributed. The whole strip is broken up in parts and in each part there are generated as many random centers as there are uniformly distributed counts. In the case, when the count in one cell is significantly higher/lower than in the neighboring cells, the randomly generated number of centers is equal to the observed count. For a given interval $\Delta l$ the galactic longitudes are purely randomly distributed, but the latitudes are generated according to

$$
b= \begin{cases}\pi / 2-\cos ^{-1}\left(1-\xi_{k}\right), & b>0^{\circ}, \\ \cos ^{-1}\left(1-\xi_{k}\right)-\pi / 2, & b<0^{\circ}\end{cases}
$$

where $\xi_{k}$ are uniformly distributed random numbers in the interval $[0,1)$. It is thus taken into account that the longitude circles approach the poles.

Afterwards a distance function $N(R)$, which is the number of centers up to a given $R$, is introduced. This function is approximated with several (5 to 15$)$ linear regressions, and random distances according to the regressions are generated. For example for Karachentsev's sample of isolated pairs $\left(b>0^{\circ}\right)$ from $R=54.5$ to $60.3 \mathrm{Mpc}$ (for $\mathrm{N}=143$ to 159 ), the 
distance function is

$$
N(R)=-20.06+2.97 R
$$

Therefore for this regression line 17 distances are generated according to $R=54.5+5.72 \xi_{k}$. Thus the random catalogs (usually 100) are generated separately for the Northern and Southern galactic hemispheres.

There are many ways to determine the two-point spatial correlation functions (e.g. Davis and Peebles 1983; Blanchard and Alimi 1988, Borner et al. 1989). The described method for calculating the correlation functions for the galaxies from the CfA survey, and CfA slice survey, for clusters of galaxies, for superclusters and for quasars were tested by the authors. The results do not differ substantually from other calculations (Davis et al. 1988; Borner and Mo 1990; de Lapparent et al. 1988; Kalinkov and Kuneva 1985, 1986; Bahcall 1988). The results for quasars especially are very close to those obtained with the so called scrambling method (Osmer 1981), but without the weakness of this technique, as demonstrated by Anderson et al. (1988).

\section{RESULTS}

(i) The Karachentsev Sample

Karachentsev's catalog $(1972,1987)$ of isolated pairs of galaxies contains 603 objects. We excluded the 18 non-pairs and all pairs having $\Delta V>1000 \mathrm{~km} / \mathrm{s}$, and $|b|<20^{\circ}$. Thus in the NGH and SGH there are 363 and 127 isolated pairs respectively. The correlation function is given in Table 1 , where the statistical uncertainty is $\Delta \xi=\left[(1+\xi) / n_{r}\right]^{1 / 2}$ and $\xi_{p p}{ }^{W}$ is the function weighted for the NGH and SGH. This two-point pair-pair correlation function is in no way related to the function shown in Fig. 44 of Karachentsev (1987), which is approximated by $\xi=36 / r, 0.7<r<8 \mathrm{Mpc}$ (for $\mathrm{H}_{0}=75 \mathrm{~km} \mathrm{~s}^{-1} \mathrm{Mpc}^{-1}$ ). For the first 4 points from Table 1 one gets $\xi_{p p}^{\mathrm{W}}=1.78-0.24 r, r<7 \mathrm{Mpc}\left(\mathrm{H}_{0}=100 \mathrm{~km} \mathrm{~s}^{-1}\right.$ $\left.\mathrm{Mpc}^{-1}\right)$.

\begin{tabular}{cccccc}
\multicolumn{7}{c}{ TABLE 1 } \\
$r(\mathrm{Mpc})$ & $\xi_{p p}^{\mathrm{N}}$ & $\Delta \xi$ & $\xi_{p p}^{\mathrm{N}}$ & $\Delta \xi$ & $\xi_{p p}^{\mathrm{W}}$ \\
& & & & & \\
1 & 1.52 & 0.38 & 1.73 & 1.57 & 1.57 \\
3 & 1.00 & 0.16 & 1.10 & 0.53 & 1.03 \\
5 & 0.48 & 0.09 & 0.84 & 0.32 & 0.57 \\
7 & 0.18 & 0.07 & -0.02 & 0.18 & 0.13 \\
9 & 0.06 & 0.06 & 0.07 & 0.16 & 0.06 \\
11 & -0.09 & 0.05 & 0.01 & $0: 13$ & -0.06 \\
13 & -0.16 & 0.04 & -0.11 & 0.11 & -0.15
\end{tabular}

It is important to note that some centers of pairs in Karachentsev's sample are located very closely. The distance between $\mathrm{K} 302$ and $\mathrm{K} 310$ is only $0.2 \mathrm{Mpc}$; for K255 and K263 it is 0.5 ; for $\mathrm{K} 133$ and $\mathrm{K} 218-0.6 \mathrm{Mpc}$ and so on. Altogether 75 mutual distances are smaller than $2.5 \mathrm{Mpc}$. 
(ii) The Hickson Sample

There are 100 compact groups of galaxies in this catalog (Hickson 1982). The redshifts of 96 groups are given by Hickson et al. (1988). We have calibrated the redshifts, using the magnitudes from Hickson et al. (1989). The linear regressions are

$$
\log z=\left\{\begin{array}{l}
-4.2399+0.1747 \mathrm{~B}_{T C} \\
-4.3463+0.1730 \mathrm{~B}_{I}
\end{array}\right.
$$

where $\mathrm{B}_{T C}$ is the asymptotic magnitude corrected for internal and external extinction and $\mathrm{B}_{I}$ is the magnitude within the $m_{B}=24.5 \mathrm{mag} \mathrm{arcsec}-2$ isophote. For Hickson groups 27, 36,41 and 50 we obtained redshifts $0.0554,0.0373,0.0291$ and 0.1172 respectively. After rejection of the groups with $|b|<25^{\circ}$ and of the most distant group (H50), there remain 50 and 45 groups in the NGH and SGH respectively. There is insufficient statistics for scales smaller than $10 \mathrm{Mpc}$. From the two-point group-group correlation function (Table 2) we might conclude that the amplitude for the NGH is very small and for the SGH it is zero. Since $\Delta \xi$ is not a standard deviation but a simple statistical uncertainty, it might turn out the $\xi_{\text {grgr }}^{\mathrm{N}}>0$ for $r<30 \mathrm{Mpc}$.

TABLE 2

$\begin{array}{ccccc}r, \mathrm{Mpc} & \xi_{\text {grgr }}^{\mathrm{N}} & \Delta \xi & \xi_{\text {grgr }}^{\mathrm{S}} & \Delta \xi \\ & & & & \\ 5 & 0.48 & 0.74 & -0.05 & 0.48 \\ 15 & 0.22 & 0.31 & -0.08 & 0.20 \\ 25 & 0.28 & 0.21 & 0.06 & 0.15 \\ 35 & -0.06 & 0.14 & -0.10 & 0.11\end{array}$

(iii) The Huchra-Geller Sample

This catalog (Huchra and Geller 1982) contains 92 groups, found in the whole-sky catalog of galaxies brighter than $M_{B}=13.2$, using a general search algorithm. There are 58 and 30 groups in the NGH and SGH after excluding 4 groups with $|b|<20^{\circ}$. It may be seen (Table 3) that $\xi^{\mathrm{N}}>0$ for $r<12 \mathrm{Mpc}$ and $\xi^{\mathrm{S}}>0$ for $r<4 \mathrm{Mpc}$.

\section{TABLE 3}

$\begin{array}{cccccc}r, \mathrm{Mpc} & \xi_{\text {grgr }}^{\mathrm{N}} & \Delta \xi & \xi_{\text {grgr }}^{\mathrm{S}} & \Delta \xi & \xi_{\text {grgr }}^{\mathrm{W}} \\ 2 & 1.33 & 0.62 & 0.46 & 0.40 & 1.03 \\ 5 & 0.40 & 0.19 & -0.22 & 0.14 & 0.19 \\ 8 & 0.16 & 0.12 & 0.01 & 0.13 & 0.11 \\ 11 & 0.17 & 0.09 & -0.06 & 0.13 & 0.09 \\ 14 & 0.07 & 0.08 & 0.18 & 0.16 & 0.11 \\ 17 & -0.11 & 0.06 & -0.05 & 0.16 & -0.09 \\ 20 & 0.02 & 0.07 & -0.17 & 0.16 & -0.04\end{array}$


(iv) The Geller-Huchra Sample

There are 176 groups in this catalog (Geller and Huchra 1983) - 123 and 53 in the NGH and SGH, respectively. They are found with the general searching, algorithm of Huchra-Geller among the galaxies in the CfA survey (Huchra et al. 1983). Table 4 shows that $\xi^{\mathrm{N}}{ }_{\text {grgr }}>0$ for $r<8 \mathrm{Mpc}$; it may well be that a small amplitude of 0.05 is preserved as far as $40 \mathrm{Mpc}$. Possibly $\xi_{\text {grgr }}^{\mathrm{S}}>0$ up to $8 \mathrm{Mpc}$.

\section{TABLE 4}

$\begin{array}{ccccccccccc}r, \mathrm{Mpc} & \xi_{\text {grgr }}^{\mathrm{N}} & \Delta \xi & r, \mathrm{Mpc} & \xi_{\text {grgr }}^{\mathrm{S}} & \Delta \xi & r, \mathrm{Mpc} & \xi_{\text {grgr }}^{\mathrm{N}} & \Delta \xi & \xi_{\text {grgr }}^{\mathrm{S}} & \Delta \xi \\ 1 & 1.39 & 0.72 & 2 & 0.35 & 0.60 & 5 & 0.08 & 0.05 & 0.17 & 0.14 \\ 3 & 0.53 & 0.22 & 5 & 0.01 & 0.27 & 15 & -0.05 & 0.03 & -0.01 & 0.06 \\ 5 & 0.18 & 0.13 & 8 & 0.08 & 0.20 & 25 & 0.08 & 0.03 & 0.11 & 0.06 \\ 7 & 0.08 & 0.10 & 11 & 0.43 & 0.18 & 35 & 0.05 & 0.03 & -0.05 & 0.05 \\ 9 & -0.06 & 0.08 & 14 & -0.07 & 0.13 & 45 & -0.06 & 0.03 & -0.16 & 0.06 \\ 11 & -0.12 & 0.07 & & & & 55 & 0.02 & 0.03 & & \\ & & & & & & 65 & -0.03 & 0.03 & & \end{array}$

(v) The Vennik Sample

There are 126 groups and subgroups in this catalog (Vennik 1984) with a depth of $3500 \mathrm{~km} / \mathrm{s}$. We excluded 4 objects -3 for having $|b|<20^{\circ}$ and the first object V1A (the Milky Way group). The amplitude of $\xi_{\text {grgr }}^{\mathrm{S}}$ is not significantly different from zero and $\xi^{\mathrm{N}}>0$ for $r<6-7 \mathrm{Mpc}$ (Table 5).

\section{TABLE 5}

$\begin{array}{cccccc}r, \mathrm{Mpc} & \xi_{\text {grgr }}^{\mathrm{N}} & \Delta \xi & r, \mathrm{Mpc} & \xi_{\text {grgr }}^{\mathrm{N}} & \Delta \xi \\ 1 & 1.77 & 0.77 & 6 & 0.22 & 0.08 \\ 2 & 1.77 & 0.35 & 7 & 0.06 & 0.07 \\ 3 & 0.58 & 0.17 & 8 & -0.02 & 0.06 \\ 4 & 0.38 & 0.12 & 9 & -0.06 & 0.06 \\ 5 & 0.06 & 0.08 & 10 & -0.25 & 0.05\end{array}$

(vi) The Ramella-Geller-Huchra Sample

The statistical sample of 95 groups (Ramella et al. 1989), found with the general searching algorithm of HG in the deeper CfA survey - the slice is used. It is evident (Table $6)$ that $\xi>0$ up to $r=20 \mathrm{Mpc}$. 
TABLE 6

$r, \mathrm{Mpc} \xi_{g r g r} \Delta \xi$

$\begin{array}{lll}2.51 & 2.26 & 0.50\end{array}$

$\begin{array}{lll}7.51 & 1.17 & 0.19\end{array}$

$\begin{array}{lll}12.51 & 0.12 & 0.10\end{array}$

$\begin{array}{lll}17.51 & 0.17 & 0.08\end{array}$

$\begin{array}{lll}22.51 & 0.10 & 0.08\end{array}$

$\begin{array}{lll}27.51 & -0.19 & 0.06\end{array}$

$\begin{array}{lll}32.51 & -0.22 & 0.05\end{array}$

(vii) The Maia-da Costa-Latham Sample

This catalog (Maia et al. 1989) contains 92 groups of galaxies identified in the Southern galactic gap (da Costa et al. 1988). The group finding algorithm of HG was used. The amplitude of the correlation function (Table 7) is positive up to $13 \mathrm{Mpc}$.

(viii) The New CfA Group Sample

There are 161 groups of galaxies found in the CfA survey in the NGH for V>600 $\mathrm{km} / \mathrm{s}$ using an algorithm developed by Kuneva and Kalinkov (1990). The amplitude of the correlation function is positive up to $6 \mathrm{Mpc}$. The statistical properties of this catalog do not differ from the previous seven catalogs.

\begin{tabular}{cccccc} 
& TABLE 7 & \multicolumn{3}{c}{ TABLE 8} \\
$r, \mathrm{Mpc}$ & $\xi_{\text {grgr }}$ & $\Delta \xi$ & $r, \mathrm{Mpc}$ & $\xi_{\text {grgr }}$ & $\Delta \xi$ \\
1 & 2.49 & 0.90 & $1-2$ & 0.38 & 0.17 \\
3 & 1.32 & 0.32 & 3 & 0.34 & 0.12 \\
5 & 0.39 & 0.17 & 4 & 0.41 & 0.10 \\
7 & 0.02 & 0.12 & 5 & 0.14 & 0.08 \\
9 & 0.17 & 0.11 & 6 & 0.07 & 0.07 \\
11 & 0.09 & 0.10 & 7 & -0.05 & 0.06 \\
13 & 0.02 & 0.10 & 8 & -0.07 & 0.05 \\
15 & -0.10 & 0.09 & 9 & -0.16 & 0.05 \\
17 & -0.25 & 0.08 & & &
\end{tabular}

\section{DISCUSSION}

From general considerations it might be expected that $\xi_{\text {grgr }}$ will have amplitudes higher than $\xi_{p p}$. Since the amplitudes of the cluster-cluster correlation function are higher than those of the galaxy-galaxy correlation function (Bahcall 1988), it could be concluded that

$$
\xi_{g g}<\xi_{p p}<\xi_{g r g r}<\xi_{c c} .
$$

Hamilton and Gott (1988) pointed out that existing measurements of three-point and fourpoint correlation amplitudes imply that pairs and triples are more clustered than galaxies. 
According to Groth and Peebles (1977) and Davis and Peebles (1983)

$$
\xi_{g r g r}(r)=20 r^{-1.8}=(r / 5)^{-1.8}, r<20 h^{-1} \mathrm{Mpc} .
$$

and neither amplitude of $\xi_{p p}$ and $\xi_{g r g r}$ in Tables 1-8 exceeds $\xi_{g g}$.

It seems that an explanation for this unexpected result might be found in two directions: (a) The estimates of $\xi_{p p}$ and $\xi_{g r g r}$ are biased and other methods of measurements have to be applied. (b) The existing samples of pairs and groups of galaxies are not fair samples (Peebles 1973).

Acknowledgement is due to D. Stefanova and H. Macrelov for their help. This work is supported in part by the Bulgarian Committee for Science (Contract 943).

\section{REFERENCES}

Anderson, N., Kunth, D., and Sargent, W. L. W. 1988, A.J., 95, 644.

Bahcall, N. A. 1988, Ann.Rev.Astr.Ap., 26, 631.

Blanchard, A., and Alimi, J.-M. 1988, Astr.Ap., 203, L1.

Borner, G., and Mo, H. J. 1990, Astr. Ap., 227, 324.

Borner, G., Mo, H., and Zhou, Y. 1989, Astr.Ap., 221, 191.

da Costa, L. N., Pellegrini, P. S., Sargent, W. L., Tonry, J., Davis, M., Meiksin, A., Latham, D. W., Menzies, J. W., and Coulson, I. A. 1988, Ap.J., 327, 544.

Davis, M., Meiksin, A., Strauss, M. A., da Costa, L. N., and Yahil, A. 1988, Ap.J.Letters, 333, L9.

Davis, M., and Peebles, P. J. E. 1983, Ap.J., 267, 465.

Geller, M. J., and Huchra, J. P. 1983, Ap.J.Suppl., 52, 61.

Groth, E., and Peebles, P. J. E. 1977, Ap.J., 217, 385.

Hamilton, A. J. S., and Gott, J. R. 1988, Ap.J., 331, 641.

Hickson, P. 1982, Ap.J., 255, 382.

Hickson, P., Kindl, E., and Auman, J. R. 1989, Ap.J.Suppl., 70, 687.

Hickson, P., Kindl, E., and Huchra, J. P. 1988, Ap.J., 331, 64.

Huchra, J., Davis, M., Latham, D., and Tonry, J. 1983, Ap.J.Suppl., 52, 89.

Huchra, J.P., and Geller, M. J. 1982, Ap.J., 257, 423.

Kalinkov, M., and Kuneva, I. 1985, Astr.Nach., 306, 283.

-. 1986, M.N.R.A.S., 218, 49p.

Karachentsev, I. D. 1972, Comm. Spets. Ap. Obs. USSR, 7, 3.

-. 1987, Dvoinye Galaktiki (Moscow: Nauka).

Kuneva, I. and Kalinkov, M. 1990, this volume.

de Lapparent, V., Geller, M. J., and Huchra, J. P. 1988, Ap.J., 332, 44.

Maia, M. A. G., da Costa, L. N., and Latham, D. W. 1989,Ap.J.Suppl., 69, 809.

Osmer, P. S. 1981, Ap.J., 247, 762.

Peebles, P. J. E. 1973, Ap.J., 185, 413.

Ramella, M., Geller, M.J., and Huchra, J.P. 1989, Ap.J., 344, 57.

Vennik, J. 1984, Tartu Astr. Obs. Teated, No.73, p.3. 


\section{DISCUSSION}

Hickson: Very few of the groups that I found occur in rich clusters of galaxies, presumably because of the isolation criterion that I used. The correlation function of these groups may also be strongly affected by the selection criteria.

Kalinkov: You are quite right. I agree with you. 